\title{
MEK inhibition enhances efficacy of bacillus Calmette-Guérin on bladder cancer cells by reducing release of Toll-like receptor 2-activated antimicrobial peptides
}

\author{
Young Mi Whang ${ }^{1, *}$, Su Bin Jin ${ }^{1, *}$, Serk In Park ${ }^{2,3, * *}$ and In Ho Chang ${ }^{1, * *}$ \\ ${ }^{1}$ Department of Urology, Chung-Ang University College of Medicine, Seoul, Korea \\ ${ }^{2}$ Department of Biochemistry and Molecular Biology and BK21 Plus Program, Korea University College of Medicine, Seoul, \\ Korea \\ ${ }^{3}$ Department of Medicine, Vanderbilt University School of Medicine, Nashville, TN, USA \\ *Young Mi Whang and Su Bin Jin contributed equally to this work \\ *** In Ho Chang and Serk In Park contributed equally to this work \\ Correspondence to: In Ho Chang, email: caucih@cau.ac.kr \\ Serk In Park, email: serkin@korea.edu \\ Keywords: bacillus Calmette-Guérin (BCG), Toll-like receptors 2 and 4 (TLR2 and TLR4), antimicrobial peptides (AMPS), MEK \\ inhibitors, bladder cancer cells \\ Received: November 30, $2016 \quad$ Accepted: May 12, $2017 \quad$ Published: May 26, 2017 \\ Copyright: Whang et al. This is an open-access article distributed under the terms of the Creative Commons Attribution License \\ 3.0 (CC BY 3.0), which permits unrestricted use, distribution, and reproduction in any medium, provided the original author and \\ source are credited.
}

\section{ABSTRACT}

Bacillus Calmette-Guérin (BCG) is one of the standard treatment options for nonmuscle-invasive bladder cancer. The details of the biological defense mechanisms against BCG remain unclear. Here, we investigated whether BCG-induced release of antimicrobial peptides (AMPs; e.g., human $\beta$-defensin-2, -3 , and cathelicidin) is involved with mitogen-activated protein kinase (MAPK) pathways, and investigated the enhanced anticancer effect of BCG through the down-regulation of Toll-like receptors (TLRs) and MAPK pathways in bladder cancer cells. BCG-infected bladder cancer cells produced AMPs as a defense mechanism against BCG, which were reduced by MEK inhibitors by blocking phosphorylation of extracellular signal-regulated kinase (ERK1/2 or MEK) and c-Jun. MEK inhibitors enhanced inhibition of bladder cancer cell growth by decreased binding of c-Jun, p65 and Pol II to the activated protein-1 promoter. Knockdown of TLR2 and TLR4 reduced ERK phosphorylation. Knockdown of TLR 2 decreased release of AMPs, which was similar to the efficacy of MEK inhibitor on BCG-infected cells. BCG-infected bladder cancer cells were more prone to induction of AMP release following TLR2 activation via ERK and c-Jun pathway mediators. In conclusion, our data suggest that the BCG-induced release of AMPs in bladder cancer cells is a promising molecular target for enhancing the immunotherapeutic efficacy of BCG in bladder cancer patients.

\section{INTRODUCTION}

Bacillus Calmette-Guérin (BCG) is a potent vaccine for Mycobacterium tuberculosis. BCG is one of the most successful immunotherapeutics for cancer, and is included in the standard treatment regimens for highrisk non-muscle-invasive bladder cancer patients [1]. However, BCG is less effective for recurrent or advanced- stage bladder cancer patients [2]. To improve the efficacy of $\mathrm{BCG}$, inhibition of pro-inflammatory mediators in the innate and acquired immune responses against mycobacteria must be clearly understood $[3,4]$.

During initial recognition of pathogens like BCG mycobacteria, Toll-like receptors 2 and 4 (TLR2 and TLR4, respectively) are activated to elicit immune responses [5]. Activation of TLRs releases antimicrobial 
peptides (AMPs) and pro-inflammatory cytokines via nuclear factor- $\mathrm{KB}(\mathrm{NF}-\mathrm{\kappa B})$ pathways $[6,7]$ and mitogenactivated protein kinases (MAPK) pathways, leading to modulation of transcription of inflammatory genes $[8,9]$. The association of TLR2 with TLR1/TLR6 or TLR4 in turn recruits different adaptor proteins allowing specific signaling cascades and gene activation programs, which contribute to resistance against mycobacteria [10]. Accordingly, mycobacterial components act as TLR agonists and elicit the release of pro- or anti-inflammatory cytokines [11].

Mammalian cells secrete numerous AMPs, such as human beta-defensins (HBD-1 or HBD-4) in epithelium and leukocytes, and cathelicidin (CAMP) in neutrophils and epithelia [12]. Mycobacteria trigger epithelial cells to express AMPs. AMPs have nonspecific cytotoxicity against a wide range of normal and malignant targets, and direct lyse mycobacteria by permeablizing the cellular membranes $[13,14]$. BCG induces HBD-2 mRNA expression in human lung epithelial cells, which exhibits antimicrobial activity against $\mathrm{BCG}$ despite up-regulated tumor necrosis factoralpha (TNF- $\alpha$ ) production by BCG-infected cell [15]. Furthermore, BCG-induced HBD-2 expression affects the internalization rate of $\mathrm{BCG}$ in bladder cancer cells, whereas the anti-HBD-2 antibody prevents the effect of HBD-2 on BCG internalization in bladder cancer cells [16]. Therefore, modulation of anti-mycobacterial activity against BCG, such as TLR downstream signaling pathways, will potentially lead to new therapeutic strategies in mycobacteria treatment.

MAPK pathways are crucial to mycobacteriainduced macrophage signaling via TLRs $[8,9]$. Similar to the molecular mechanisms by which mycobacteria upregulates AMPs in epithelial cells, MAPK pathway activation contributes to the regulation of inflammatory processes in BCG-infected epithelial cells. HBD-2 participates in anti-bactericidal activities directed against $\mathrm{BCG}$, which is mediated by MAPK signaling pathways regulating HBD-2 expression in human epithelial cells during BCG infection [17]. Furthermore, M. bovis BCG-mediated TLR2 signaling triggers the production of nitric oxide, which negatively regulates interferongamma (IFN- $\gamma$ )-induced immune gene expression for macrophages [18].

The present study demonstrates that MEK inhibitors enhance BCG treatment-induced tumor cell death via the blockage of AMPs release. The enhanced antitumor effects of BCG in bladder cancer cells are associated with the inhibition of TLR2-medated MEK pathway. The findings implicate the activation of intracellular signaling pathways in response to $\mathrm{BCG}$ infection as a novel strategy to boost BCG treatment efficacy in urothelial carcinomas.

\section{RESULTS}

\section{BCG stimulates release of AMPs and induce ERK (1/2) phosphorylation in bladder cancer cells}

To determine the effect of BCG-induced AMPs release on bladder cancer cells, the cells were treated with 10 MOI BCG for 8 hours, followed by ELISA quantification of AMPs. BCG stimulated the release of HBD-2 and -3 by 3 -fold compared to untreated control in both types of bladder cancer cells. The CAMP level was increased by over 8-10-fold in BCG-treating bladder cancer cells compared to untreated cells (Figure 1A). We hypothesized that BCG-induced expression of inflammatory mediators, including chemokines and AMPs, is associated with the MAPK signaling pathway. Previous reports showed that BCG activates the MAPK and phosphoinositol-3 kinase pathways as signaling events leading to pro-inflammatory gene expression $[19,20]$. Therefore, we determined whether BCGdependent activation of MAPK pathway can be blocked by MAPK-specific inhibitors in bladder cancer cells. ERK phosphorylation was induced by BCG treatment in both 5637 and T24 cells (Figure 1B) and the effect was completely blocked by MEK inhibitor in both 5637 and T24 cells. JNK inhibitors also blocked phosphorylation of JNK only in T24 cells (Figure 1C). These results suggest that BCG treatment can stimulate release of antimicrobial peptide via phosphorylation of ERK in bladder cancer cells.

\section{BCG treatment selectively induces the MEK pathway in bladder cancer cells}

We hypothesized that inhibition of MEK pathway avoids a BCG-induced antimicrobial effect on bladder cancer cells, resulting in blockage of the release of AMPs. We next tested whether BCG treatment in bladder cancer cells in combination with the MAPK specific inhibitors U0126, PD98059, SB20358, or SP600125 more effectively inhibited tumor cell proliferation. Both T24 and 5637 cells exhibited resistance to BCG single treatment, with no growth inhibitory effect by 10 MOI BCG (Figure $2 \mathrm{~A}$ ). Then, we observed that high concentration of 30 MOI BCG induced growth inhibition by $40 \%$, which was reduced by treatment with recombinant AMPs in both T24 and 5637 cells (Figure 2B). Sensitivity to BCG was exacerbated by the U0126 and PD98059 MEK inhibitors, which inhibit MEK1/ MEK2 (U0126 inhibits both MEK1/2; PD98059 inhibits a highly selective MEK1 activation) compared with $\mathrm{BCG}$ in combination with other MAPK inhibitors (Figure 2C). These results are consistent with the involvement of the BCG-induced MEK pathway 
A
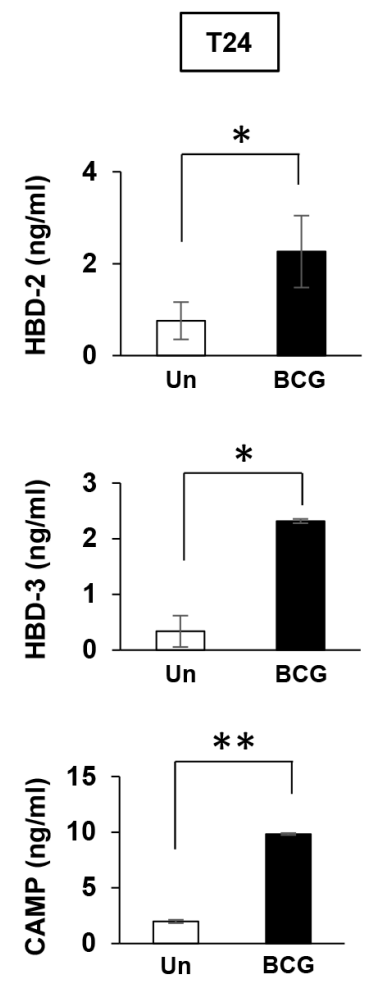

B

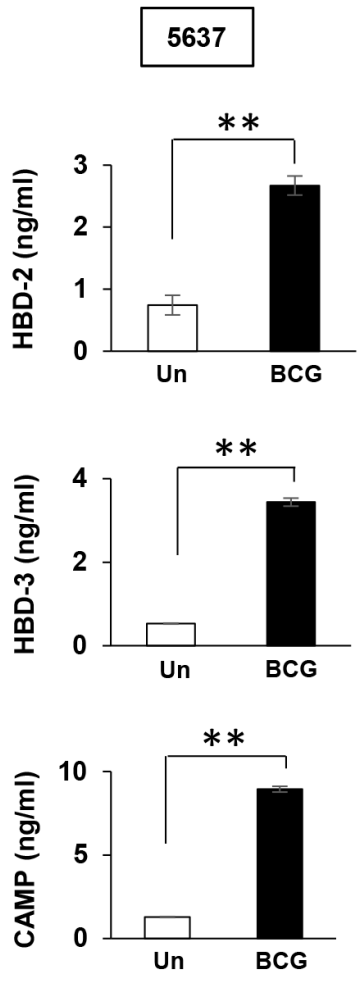

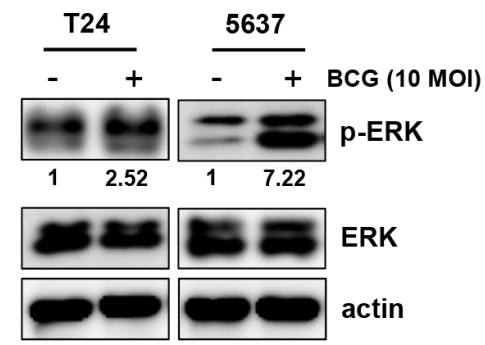

C
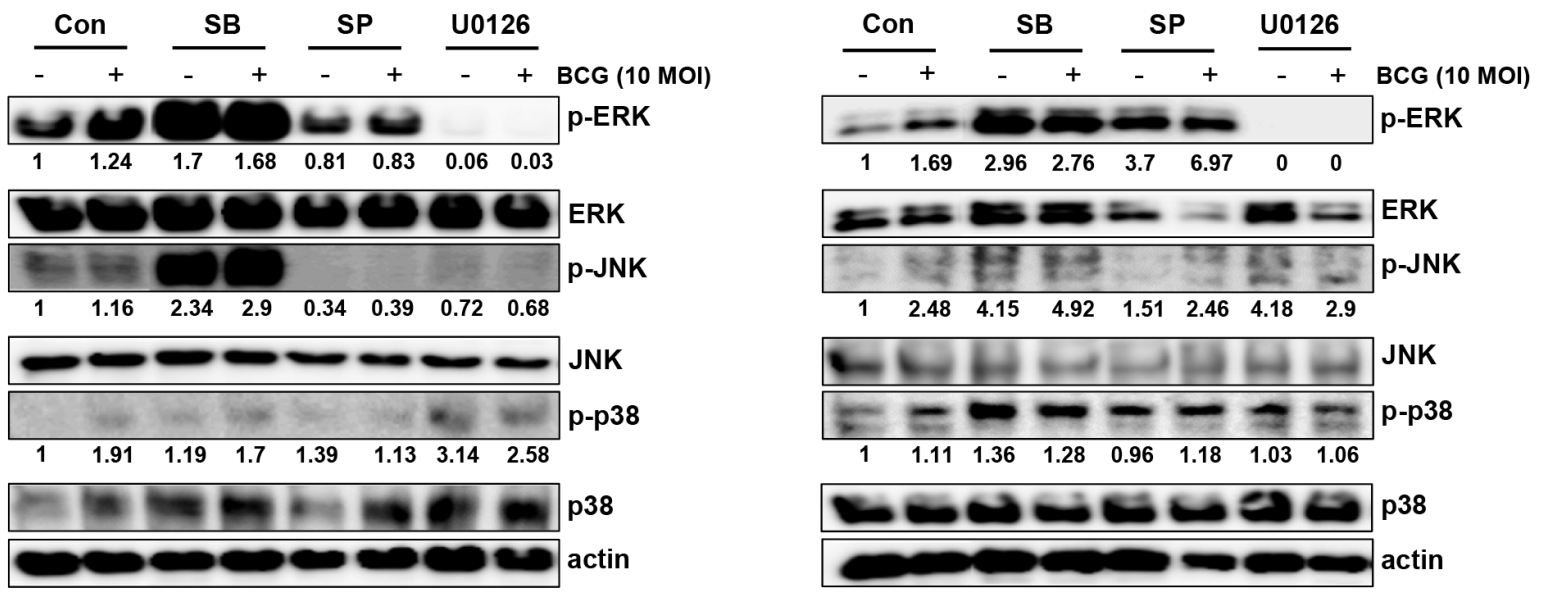

Figure 1: BCG stimulates release of antimicrobial peptides and induces ERK phosphorylation in two bladder cancer cell lines. (A) T24 and 5637 bladder cancer cells were infected with BCG (10 MOI for 8 h) or empty vector (Un; untreated), followed by ELISA of antimicrobial peptides (HBD-2, HBD-3, and CAMP) in the culture supernatant. Data are mean $\pm \mathrm{SD}$ ( $\mathrm{n}=3$ per group). * $P<0.05$ and ** $P<0.01$, Student's T-test (B) Western blotting analyses showing BCG-induced ERK phosphorylation in T24 and 5637 cells. (C) Western blotting analyses showing phosphorylation of ERK, JNK, and p38 in T24 (left panels) and 5637 (right panels) cells pre-treated with SB203580 (SB; a specific p38 inhibitor; $10 \mu \mathrm{M}$ ), SP600125 (SP; a specific JNK inhibitor; $5 \mu \mathrm{M}$ ), or U0126 (an MEK1/2 or ERK1/2specific inhibitor; $10 \mu \mathrm{M}$ ) for $2 \mathrm{~h}$, followed by BCG infection (10 MOI for $8 \mathrm{~h}$ ). DMSO was used as control (Con). Numerical data represent phospho-ERK/total ERK and phospho-JNK/total JNK ratios determined by densitometric analyses. All expression ratios were normalized to the untreated group. 
A

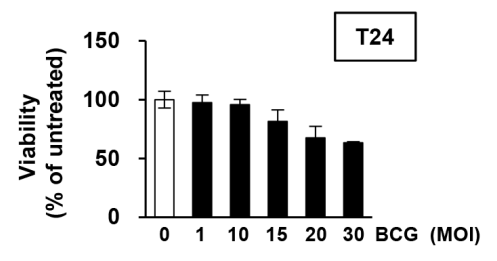

B

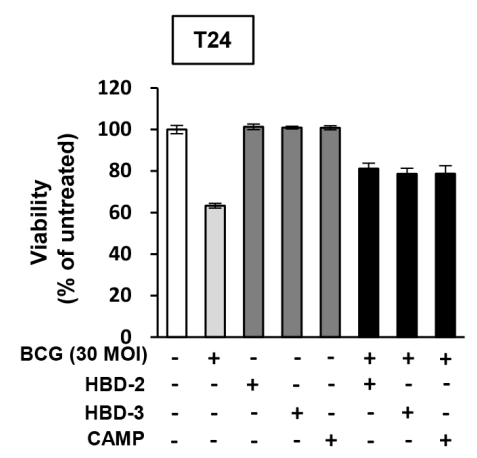

C

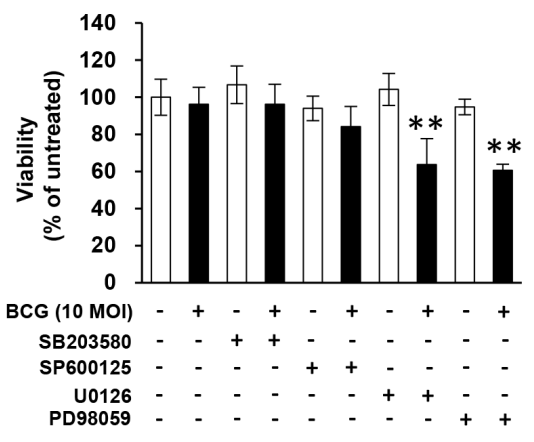

D

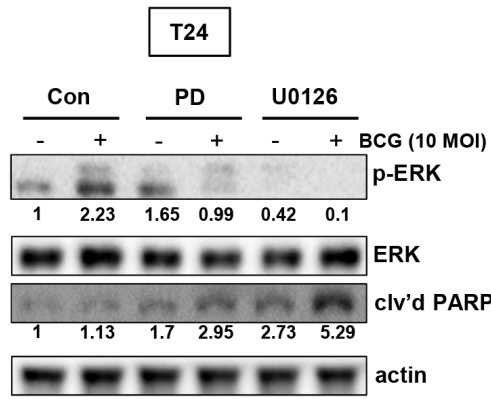

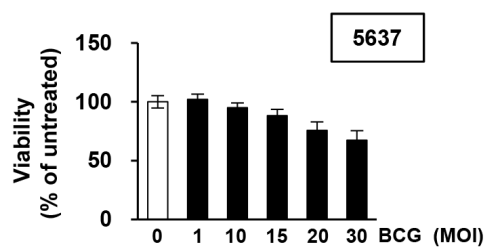

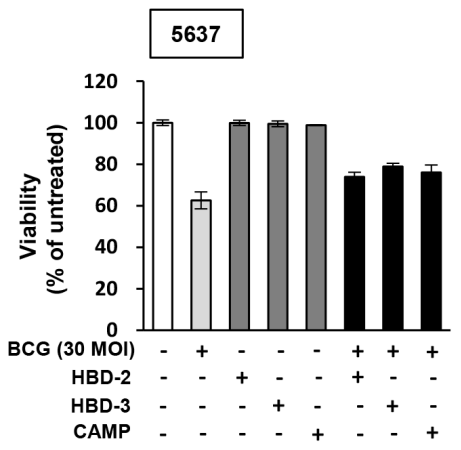

5637
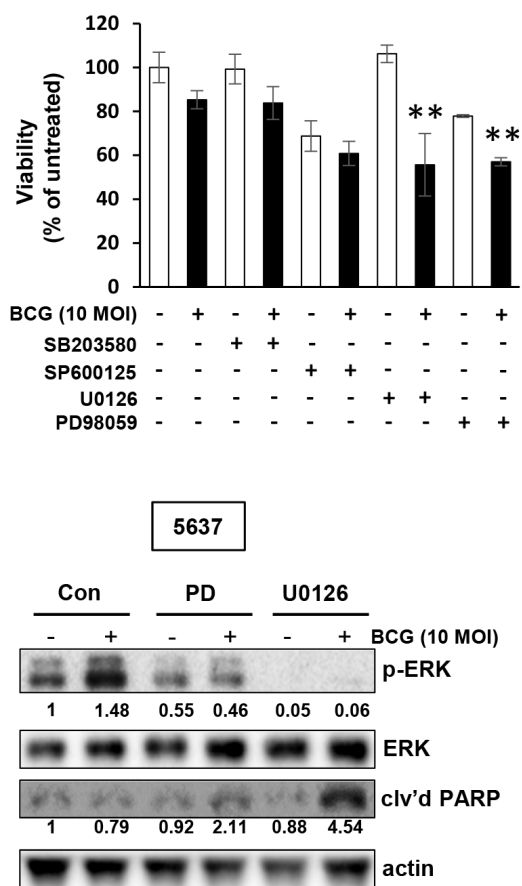

Figure 2: Pharmacological inhibition of MEK pathways increases the anti-proliferative effects of BCG in bladder cancer cells. (A) Cells were plated in 96-well plates $24 \mathrm{~h}$ before adding BCG. Viable cells were quantified by MTT assays. (B) Cells were pretreated with antimicrobial peptides (HBD-2, HBD-3, and CAMP), and subsequently infected with $30 \mathrm{MOI}$ of BCG (i.e. a dosage determined by data in panel A to induce greater than $30 \%$ growth inhibition). After 48 hours, viable cells were quantified by MTT assays. (C) Cells were pretreated with specific inhibitors of p38 (SB203580; $10 \mu \mathrm{M}$ ), JNK (SP600125; $5 \mu \mathrm{M}$ ) and/or ERK (U0126; $10 \mu \mathrm{M}$ or PD98059; $1 \mu \mathrm{M}$ ). After 2 hours, the cells were infected with $10 \mathrm{MOI}$ of BCG (i.e. sub-optimal dosage to suppress cell proliferation as shown in the panel A) for 48 hours, followed by MTT cell viability assays. Data are mean $\pm \mathrm{SD}$ ( $\mathrm{n}=6$ per group). ${ }^{*} P<0.01$, U0126/ BCG+U0126, BCG/BCG+U0126, PD98059/ BCG+PD98059, or BCG/BCG+PD98059. Student's T-test. (D) Western blotting analyses showing apoptosis by combined treatment with MEK inhibitors and BCG in T24 (left panels) and 5637 (right panels) cells pre-treated with PD98059 (a specific MEK1 inhibitor; $1 \mu \mathrm{M}$ ) or U0126 (a specific MEK1/2 inhibitor; $10 \mu \mathrm{M}$ ) for $2 \mathrm{~h}$, followed by BCG infection (10 MOI for 48 hours). DMSO was used as empty vector controls (Con). The expressions of phosphorylated ERK1/2 was calculated as the ratio of phosphorylated ERK/2 to total ERK1/2 protein expression using densitometric analyses. The ratio of cleaved PARP product to actin protein expression were calculated similarly. Expression ratios were normalized to the untreated group. 
and blockage of phosphorylated ERK by MEK inhibitors (Figure 1B and 1C). As shown in Figure 2D, treatment of BCG with MEK inhibitors abolished phosphorylation of EKR and induced cleavage of poly (ADP-ribose) polymerase (PARP), suggesting that combined treatment with BCG and MEK inhibitors induces apoptosis in BCG-treated bladder cancer cells. Therefore, inhibition of the ERK pathway could increase sensitivity to BCG by repressing BCG-induced phosphorylation of ERK and subsequent inhibition of the release of AMPs.

\section{MEK inhibitors reduce BCG-mediated transcriptional activation of AMPs in bladder cancer cells}

We first evaluated the effect of MEK inhibitors on the reduction of $\mathrm{BCG}$-induced AMPs release from the supernatants of conditioned media. As shown in Figure $3 \mathrm{~A}$, in T24 cells, BCG increased in the release of three AMPs, and this effect was suppressed by MEK inhibitors. BCG-induced HBD-2/3 and CAMP release in 5637 cells were reduced by treatment with MEK inhibitors. Given the activation of the transcription factor AP-1 is essential to HBD-2 and -3 and/or CAMP production, we sought to determine the binding of c-Jun, p65, and/or Pol II to the AP1 promoter in BCG- and/or MEK inhibitor-treated T24 cells using a ChIP assay. Phosphorylation of c-Jun was increased in BCG-treated bladder cancer cells, but BCG-induced phosphorylation of c-Jun was completely abolished by MEK inhibitor in T24 cells (Figure 3B). In 5637 cells, BCG-induced phosphorylation of c-Jun was reduced in comparison to BCG alone treatment. This effect was involved in activation of ERK phosphorylation, which was reduced by treatment with MEK inhibitors (Figure 2D). Therefore, we next tested whether c-Jun, p65, and Pol II binding to the HBD-2, -3 or CAMP promoters could be increased by BCG treatment. As shown in Figure $3 \mathrm{C}$, following treatment with MEK inhibitors, binding of c-Jun and p65 to the HBD-2 promoter was reduced in T24 cells, but the binding of Pol II was only slightly reduced. Inhibition of c-Jun binding to HBD-3 by MEK inhibitors was less efficient than p65 and Pol II, but c-Jun and p65 binding to CAMP promoter was strongly reduced by MEK inhibitor in comparison with Pol II. These data collectively indicate that $\mathrm{BCG}$ induces transcriptional activity of AMPs, which can be blocked by MEK inhibitor in bladder cancer cells, and which is potentially mediated by recruitment of AP-1 subunit c-Jun, p65, and Pol II.

\section{BCG-induced AMPs release is mediated by TLR2 in bladder cancer cells}

As an innate immune response system, the TLR family recognizes pathogens and BCG activates TLR2 and TLR4 [5]. Therefore, to investigate the role of TLR2 or TLR4 in BCG-dependent AMPs release in tumor cells, we generated stable TLR2 or TLR4 knockdown lines from T24 cells and confirmed the reduced expression of TLR2 or TLR4 by Western blotting (Figure 4A, upper panel). The stable TLR2 knockdown T24 cells exhibited lower induction of phosphorylation of ERK than nonspecific shRNA control cells (Figure 4A, lower panels). Whereas similar to the non-specific shRNA control T24 cells, stable TLR4 knockdown cells exhibited increase in phosphorylation of ERK by BCG treatment. Activated TLR2 and TLR4 initiate MAPK signaling pathways [21], which in turn activates c-Jun and release AMPs. As shown in Figure 4B, shRNA TLR2-knockdown T24 cells displayed reduced basal levels of phosphorylated c-Jun, which is downstream target of ERK, compared with non-specific shRNA control T24 cells. This effect was not observed in shRNA TLR4 knockdown cells (Figure 4B). We then evaluated the induced AMPs release from the supernatants of conditioned media in shRNA TLR2 and TLR4 knockdown cells. As shown in Figure 4C, BCG increased in the release of three AMPs in non-specific shRNA control T24 cells, but this effect was suppressed in only shRNA TRL2 knockdown cells. To confirm the effect of down-regulation of TLR2 on the release of AMPs due to the blockade of MEK-c-Jun downstream pathways, a ChIP assay was used to determine c-Jun binding to the HBD-2, HBD-3, or CAMP promoters. As shown in Figure 4D, shRNA TLR2 knockdown T24 cells showed reduced c-Jun binding to the HBD-2, HBD-3, and CAMP promoters, compared with non-specific shRNA control T24 cells. This effect was not observed in shTLR4 knockdown T24 cells. These data are consistent with our data showing decreased phosphorylation of ERK and c-Jun by MEK inhibitors leading to suppressed release of AMPs. These results suggest that initiation in response to BCG by TLR2, not by TLR4, is induced in a MEKdependent manner and is responsible for the subsequent induction of the release of AMPs.

\section{TLR2-knockdown cells are more sensitive to BCG treatment}

Finally, we confirmed that BCG inhibited the growth of TLR2-knockdown T24 cells more potently than inhibition of non-specific control cells (Figure 5A, left panel). However, in stable TLR4-knockdown T24 cells, BCG did not induce a more substantial reduction in cell proliferation compared with the vector control (Figure 5A, right panel). In addition, treatment of 30 MOI BCG highly induced cleavage of PARP in shRNA TLR2-knockdown T24 cells compared to non-specific control and shRNA TLR4-knockdown cells. BCG reduced the colony-forming ability of TLR2-knockdown cells more effectively than the non-specific control cells (Figure 5C), while this effect was not observed in TLR4-knockdown T24 cells. These results suggest that TLR2-downregulation enhances sensitivity to BCG by suppressing cell viability and colony forming ability of bladder cancer cells. 
A

T24
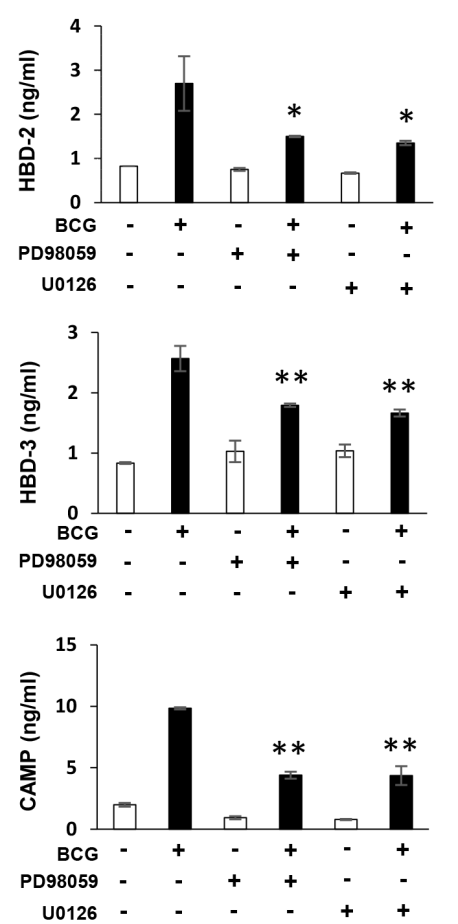

B

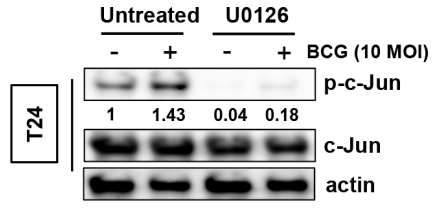

5637
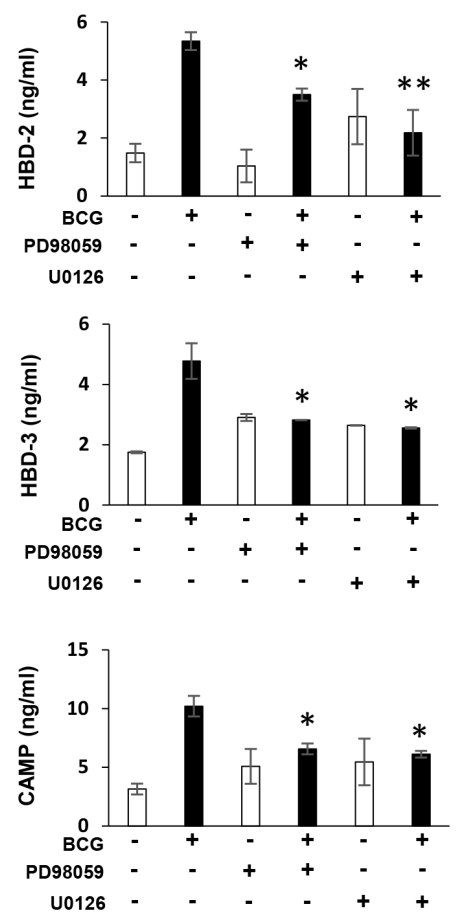

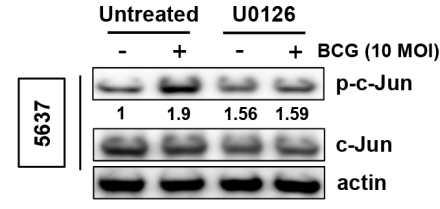

C
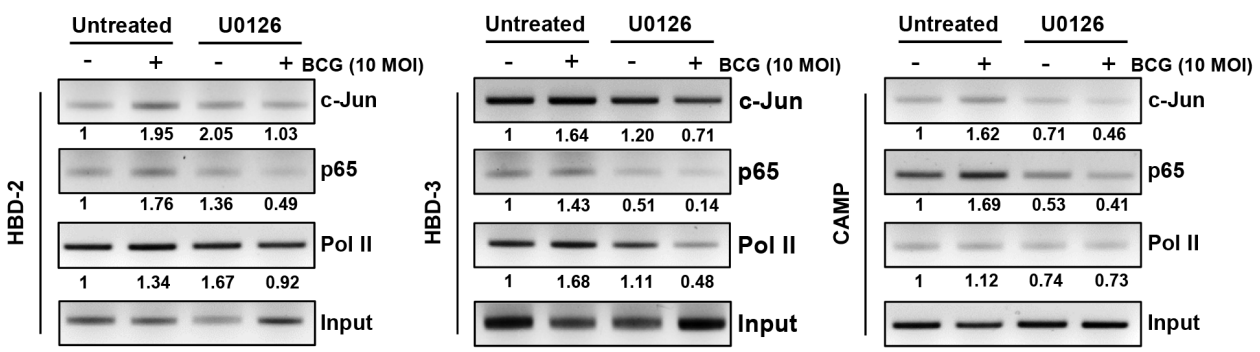

Figure 3: Inhibition of MEK pathway suppresses BCG-mediated release of AMPs in bladder cancer cells. (A) T24 (left panels) and 5637 (right panels) bladder cancer cells were pretreated with two MEK-specific inhibitors (U0126 $10 \mu$ M or PD98059 $1 \mu \mathrm{M}$ for $2 \mathrm{~h}$ ), and subsequently infected with BCG (10 MOI for $8 \mathrm{~h}$ ). Antimicrobial peptides (HBD-2, HBD-3, and CAMP) in culture supernatants were quantified by ELISA. Data are mean $\pm \mathrm{SD}$ of 3 independent experiments. ${ }^{*} P<0.05$ and $* * P<0.01, \mathrm{BCG} / \mathrm{BCG}+\mathrm{PD} 98059$ or BCG/BCG+U0126. Student's T-test. (B) Western blotting analyses showing BCG-induced c-Jun phosphorylation is suppressed by MEK inhibitors in both T-24 and 5637 cells. Cells were pretreated with an MEK-specific inhibitor (U0126; $10 \mu \mathrm{M}$, PD98059; $1 \mu \mathrm{M})$ for $2 \mathrm{~h}$. Subsequently, the cells were infected with BCG (10 MOI for $48 \mathrm{~h})$ and cell lysates were subjected to Western blot analysis. Phospho-Jun/ total Jun expression ratios were determined by densitometric analyses. Expression ratios were normalized to the untreated group. (C) Chromatin immunoprecipitation assay showing that MEK inhibition reduces BCG-mediated AMP release in bladder cancer cells. T24 cells were treated with U0126 (10 $\mu \mathrm{M}$ for 2 hours). The cells were subsequently infected with BCG (10 MOI for 8 hours) and fixed with 10\% formaldehyde. The lysates were subject to ChIP assay using anti-c-Jun, -p65, and -Pol II antibodies. Expressions of AMPs were calculated as the ratio of c-Jun, p65, and Pol II to input DNA expression using densitometric analysis. All expression ratios were normalized to the untreated group. 
A

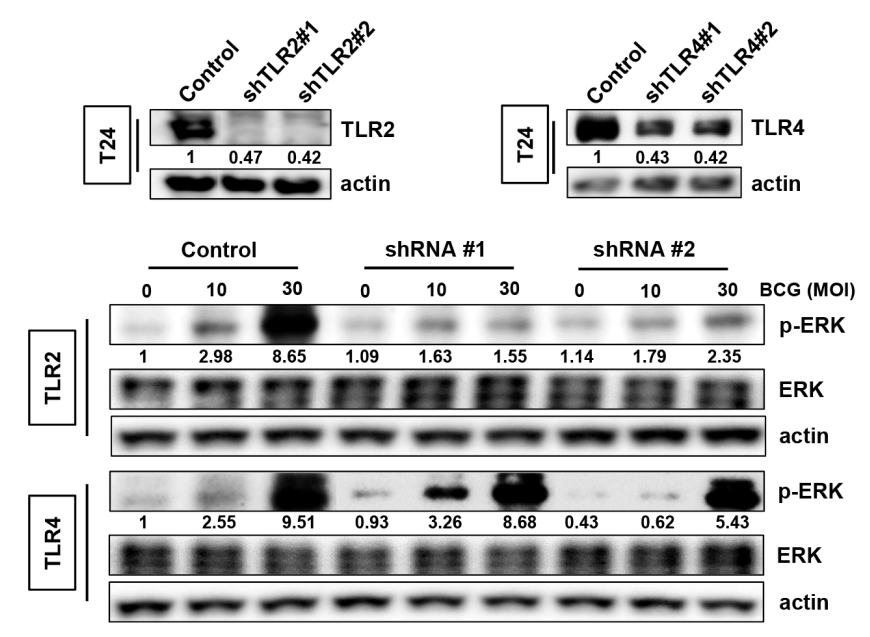

B
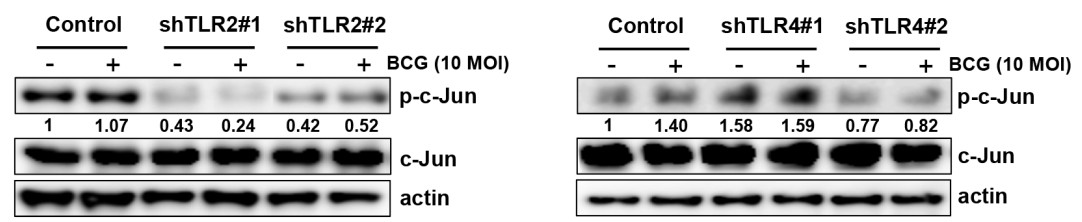

C
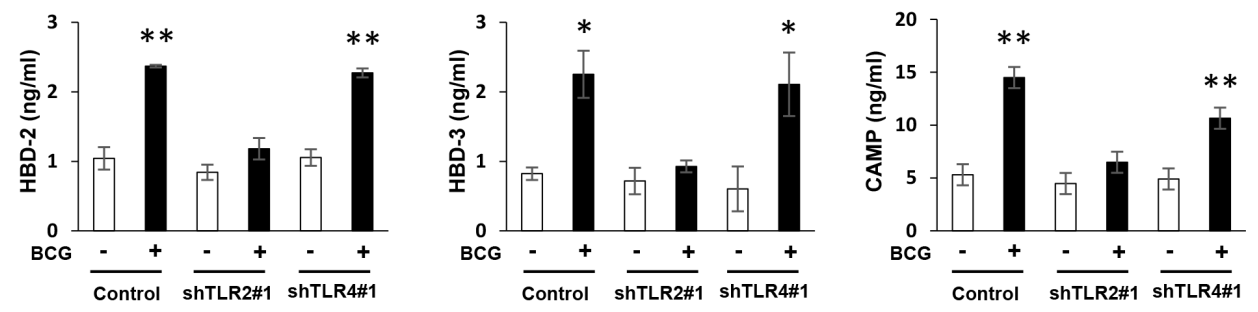

D
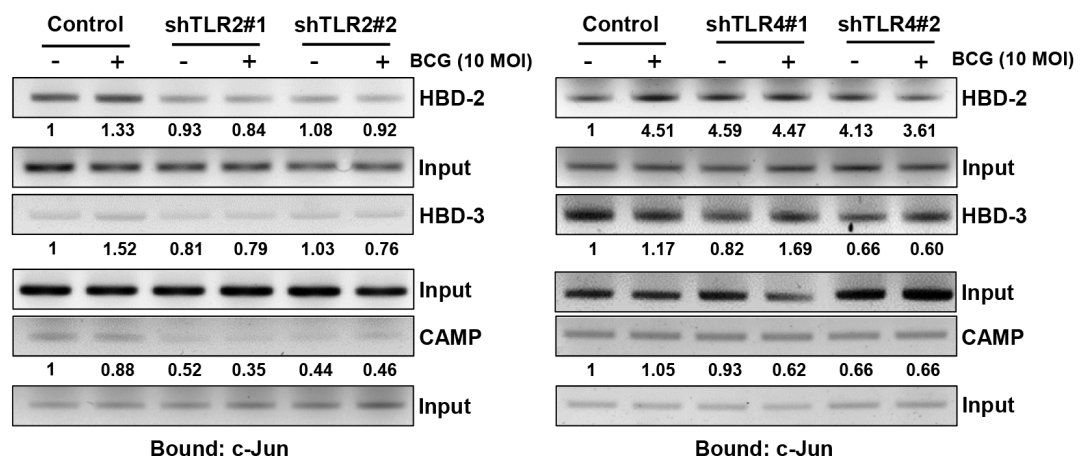

Figure 4: TLR2 participates in BCG-induced activation of MEK pathway for AMP release in bladder cancer cells. (A) Western blotting analyses demonstrating the reduction of Toll-like Receptor 2 (TLR2) or TLR4 protein expression in T24 cells transfected with two shRNA-expressing plasmids targeting TLR2 or TLR4 sequences (shTLR2\#1, shTLR2\#2, shTLR4\#1 and shTLR4\#2, respectively; upper panels). Non-specific scrambled shRNA was used as control. shRNA TLR2 or TLR4 knockdown cells were selected with puromycin, and infected with BCG (10 or $30 \mathrm{MOI}$ for $48 \mathrm{~h}$; lower panels). Phospho ERK expression was detected by Western blotting. (B) Stable clones of TLR2- or TLR4-knockdown T24 cells were treated with BCG (10 MOI for $8 \mathrm{~h})$ and analyzed for expression of phosphorylated c-Jun. Phospho-Jun/total Jun expression ratios were calculated by densitometric analysis, and indicated under each lane. Expression ratios were normalized to the untreated group. (C) StableshTLR2 or shTLR4 T24 bladder cancer cells were infected with BCG (10 MOI for 8 h), followed by ELISA of antimicrobial peptides (HBD-2, HBD-3, and CAMP) in the culture supernatant. Data are mean \pm SD ( $\mathrm{n}=3$ per group). ${ }^{*} P<0.05$ and $* * P<0.01$ Student's T-test. (D) ChIP assays using anti-c-Jun antibody to detect physical interactions between c-Jun and HBD2, HBD-3 or CAMP. TLR2-or TLR4-knockdown T24 cells were infected with BCG (10 MOI for $8 \mathrm{~h}$ ) and cross-linked with $10 \%$ formaldehyde. Expressions of AMPs were calculated as the ratio of c-Jun to Input DNA expression using densitometric analysis. All expression ratios were normalized to the untreated group. 
A
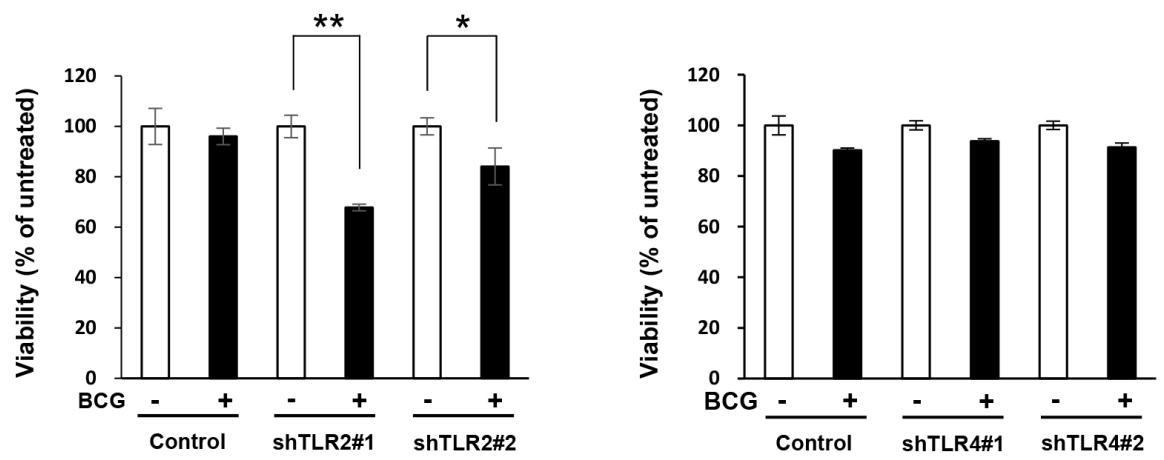

B

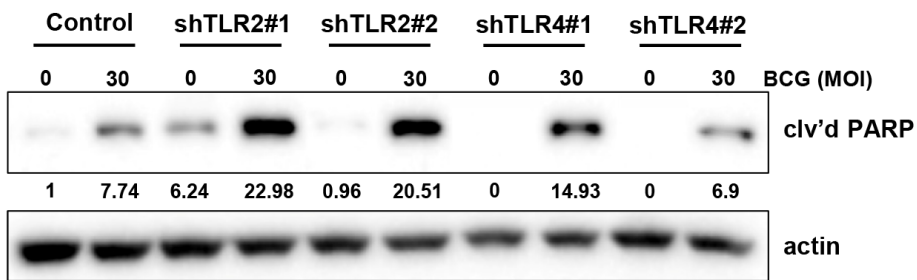

C
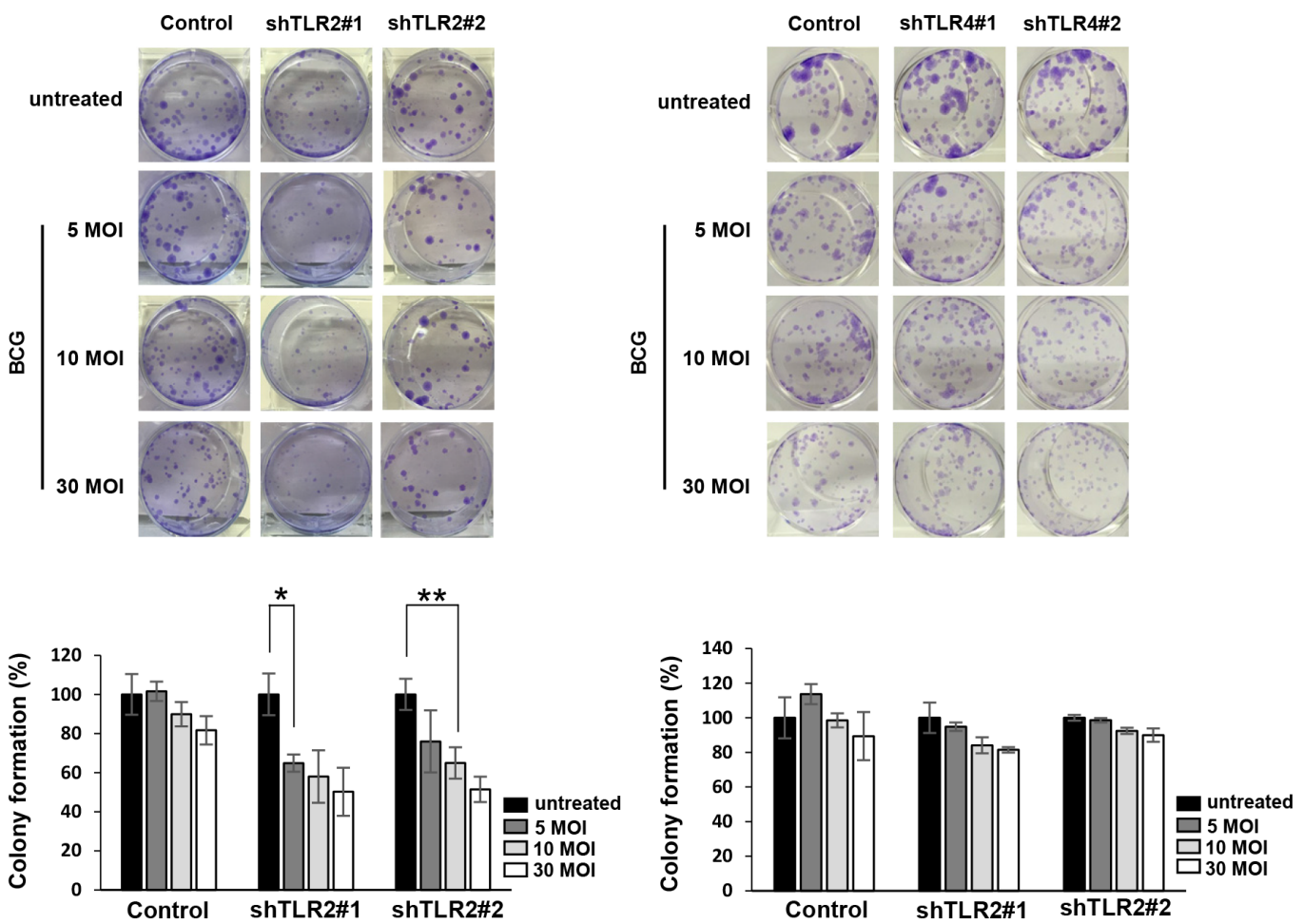

Figure 5: Inhibition of TLR2, but not TLR4, pathway enhances anti-proliferative effects of BCG in bladder cancer cells. (A) TLR2- and TLR4-knockdown T24 cells were plated in 96-well plates for $24 \mathrm{~h}$, and were infected with BCG (10 MOI for 48 h). Cell viability was determined using the MTT assay. Data are mean $\pm \mathrm{SD}(\mathrm{n}=6)$. ${ }^{* *} P<0.01$ and $* P<0.05$. Student's T-test (B) Western blotting analyses showing apoptosis by combined treatment with BCG (30 MOI) in TLR2-sable knockdown T24 cells for 72 hours. The ratio of cleaved PARP product to actin protein expression were calculated by densitometric analyses. All expression ratios were normalized to the untreated group. (C) Colony-formation assay of TLR2- or TLR4-stable knockdown T24 cells treated with 5 to 30 MOI BCG for 8 hours. Subsequently, cells were re-plated in 12-well plates at low density and allowed to grow for two weeks before fixation, crystal violet staining and counting. Data are mean $\pm \mathrm{SD}(\mathrm{n}=3) . * * P<0.01$ and $* P<0.05$ Student's T-test. 


\section{DISCUSSION}

While intravesical BCG administration has clear clinical benefits for non-muscle-invasive bladder cancer, BCG immunotherapy for treating urothelial carcinoma remains limited [22]. The precise mechanism of BCG immunotherapy, particularly downstream signaling pathways like TLR in the release of AMPs, has to be elucidated. In the present study, we demonstrated that BCG-induced MAPK signaling activation results in the release of AMPs (Figure 1A and 1B). We also demonstrated that MEK inhibitors suppressed ERK phosphorylation and decreased the viability of bladder cancer cells during BCG infection (Figures $1 \mathrm{C}$ and $2 \mathrm{C}$ ). Calcium channel-stimulated Mycobacterium tuberculosis exhibit protective responses of activated macrophages associated with inhibited generation of reactive oxygen species (ROS) generation, which is dependent on TLRMAPK pathways [23]. Our findings indicate that MEK inhibitors are beneficial to BCG-refractory bladder cancer cells. Furthermore, growth inhibition is elevated in MEKinhibited BCG-infected cancer cells, and the inhibitory effects of MEK inhibitor is enhanced by inhibited release of AMPs.

To further elucidate downstream targets of MEK inhibitor-dependent AMPs down-regulation in BCGinfected cells, we analyzed c-Jun activation and binding of c-Jun, p65, and Pol II to AMP promoters during responses to BCG. Proximal promoters of AMP genes have a consensus transcription factor AP-1, and NF- $\mathrm{kB}$ and $\mathrm{AP}-1$ are important in the regulation of AMPs in different cell types and for different stimuli [24-26]. In this study, c-Jun phosphorylation was increased after BCG-induced ERK phosphorylation (Figure 3B), which was abolished by MEK inhibition. Furthermore, MEK inhibitors obviated the recruitment of AP-1 subunit c-Jun, p65, and Pol II to AMP promoters, thereby demonstrating that the mediation of AP-1 is in part as a transcriptional factor of BCGinduced AMPs release in bladder cancer cells.

Presently, BCG induced the release of AMPs by activating ERK/c-Jun pathways in bladder cancer cells. Given the knowledge that the BCG cell wall consists of mycolic acids, arabinogalactan, and peptidoglycan, which all potentially activate TLR2 and TLR4 [5], we hypothesized that activation of TLR2 and TLR4 signaling is required in immune responses against BCG. An unexpected finding was that BCG selectively induced ERK phosphorylation and release of AMPs release only following TLR2 activation, not TLR4 activation (Figure 4A). Further analyses indicated an essential role of TLR2 for binding of c-Jun, p65, and Pol II to AMP promoters in BCG-infected bladder cancer cells (Figure 4D). The present and prior results using a reporter gene assay system and TLR knockout mice [27] suggest that purified BCG activates NF- $\kappa B$ promoters in a TLR2-dependent manner. TLR2 and $\beta 2$ integrin triggers BCG-induced macrophage apoptosis, in which MEK/ERK activation is crucial following the engagement of TLR2 [27]. However, several reports provided evidence supporting the importance of TLR4 in BCC-induced apoptosis of macrophages [28, 29]. TLR4 is reportedly not required to control acute BCG infection, and TLR4-deficient mice display reduced bacterial clearance during long-term infection depending on the types of mutation in the common adaptor protein MyD88 [10]. Therefore, differing effects of TLR2 and/ or TLR4 activation during response to BCG have been described. Presently, no significant changes were observed in the phosphorylation of ERK and c-Jun binding to AMP promoters in BCG-infected shRNA TLR4 knockdown cells in comparison with shRNA control cells, whereas a significant decrease was observed in shRNA TLR2 knockdown cells (Figure 4A and 4B). Finally, we showed that the antitumor efficacy of BCG was enhanced only in TLR2 knockdown cells compared to shRNA control cells, whereas BCG treatment had no effect on cell viability and colony formation ability in TLR4 knockdown cells (Figure 5). This efficacy was similar to ERK signaling in BCG-infected cells by MEK inhibitors. Taken together, our findings provide a rationale for BCG treatment in which the release of AMPs may be controlled by ERK phosphorylation and c-Jun activated- AP-1 transcription.

In conclusion, we demonstrate for the first time that MEK inhibitors enhance sensitivity to BCG treatment in bladder cancer cells. This furthers the understanding of the underlying mechanisms blocking TLR2-derived AMPs release (Figure 6). Although MAPK signaling is implicated in the promotion of cell survival and proliferation, BCG-induced AMPs rely more heavily on TLR2-ERK signaling for the innate and adaptive immune responses. The combination of BCG plus MEK inhibitors may be useful as a salvage regimen in BCG failures. Lowdose BCG treatment may be valuable for BCG refractory bladder cancer patients.

\section{MATERIALS AND METHODS}

\section{Cell cultures and reagents}

The 5637 and T24 human urothelial bladder cancer cell lines were purchased from the American Type Culture Collection (VA, USA). These cell lines were confirmed to be free of mycoplasma contamination using a PCRbased detection kit (Intron Biotechnology, WA, USA). Cells were maintained in complete medium containing $10 \%$ fetal bovine serum (FBS; Gibco, MD, USA) in a humidified atmosphere with $5 \% \mathrm{CO}_{2}$. To generate stable knockdown cell lines, plasmid TLR2 and TLR4 shorthairpin RNA (shRNA) constructs and a non-specific shRNA control were purchased from Qiagen (CA, USA). Cells were transfected with $5 \mu \mathrm{g}$ shRNA plasmid DNA using Lipofectamine 2000 (Invitrogen, CA, USA) according to the manufacturer's instructions. To obtain 
stable shRNA-expressing clones, 5637 or T24 cells were plated in 6-well plates and puromycin selection $(0.1-1 \mu \mathrm{g} / \mathrm{ml})$ was initiated 2 days after transfection [30]. Mycobacterium bovis BCG was obtained as a commercial lyophilized preparation (Onco-Tice, NJ, USA). BCG was resuspended in phosphate buffered saline and aliquots with a multiplicity of infection (MOI) of $100\left(1 \times 10^{7}\right.$ cells/ $\mathrm{ml})$ were prepared and stored at $-70^{\circ} \mathrm{C}$ until use. MEK inhibitors U0126 and PD98069, p38 inhibitor SB203580, and the c-Jun N-termal kinase (JNK) inhibitor SP600125 were purchased from Merck Millipore (MA, USA).

\section{Cell viability and colony formation assays}

Cells were seeded into wells of 96-well plates at $3 \times 10^{5}$ cells per well and treated with various concentrations of rapamycin. After 48 hours, cell viability was analyzed using the 3-(4,5-dimethylthiazol-2-yl)-2,5diphenyltetrazolium bromide (MTT) assay according to the manufacturer's instructions (Sigma-Aldrich, MO, USA). Colony formation assays were performed following treatment with BCG $(5,10$, and $30 \mathrm{MOI})$ for 48 hours, at which time the cells were reseeded into 12 -well plates at low density $\left(1 \times 10^{3}\right.$ cells per well $)$ in complete medium for 2 weeks [31]. Colonies were fixed with methanol and stained with $0.1 \%$ crystal violet (Sigma-Aldrich). Colony numbers were assessed visually and colonies measuring at least $50 \mu \mathrm{m}$ were counted.

\section{Quantification of HBD2, HBD3, and CAMP proteins}

Release of human $\beta$-defensin-2 and -3 (HBD2 and HBD-3, respectively) and cathelicidin (CAMP) were measured by ELISA after treatment with BCG and/or MEK inhibitors in bladder cancer cells. Cells were pretreated with MAPK inhibitors. Two hours later, BCG was added and incubated for 8 hours. The conditioned medium samples were diluted and added to the recommended incubation procedures. After stopping the reaction, the samples were measured using a VERSA max microplate reader (Molecular Devices, CA, USA).

\section{Western blotting}

Total cell lysates were resolved using sodium dodecyl sulfate-polyacrylamide gel electrophoresis and Western blotting was performed as described previously

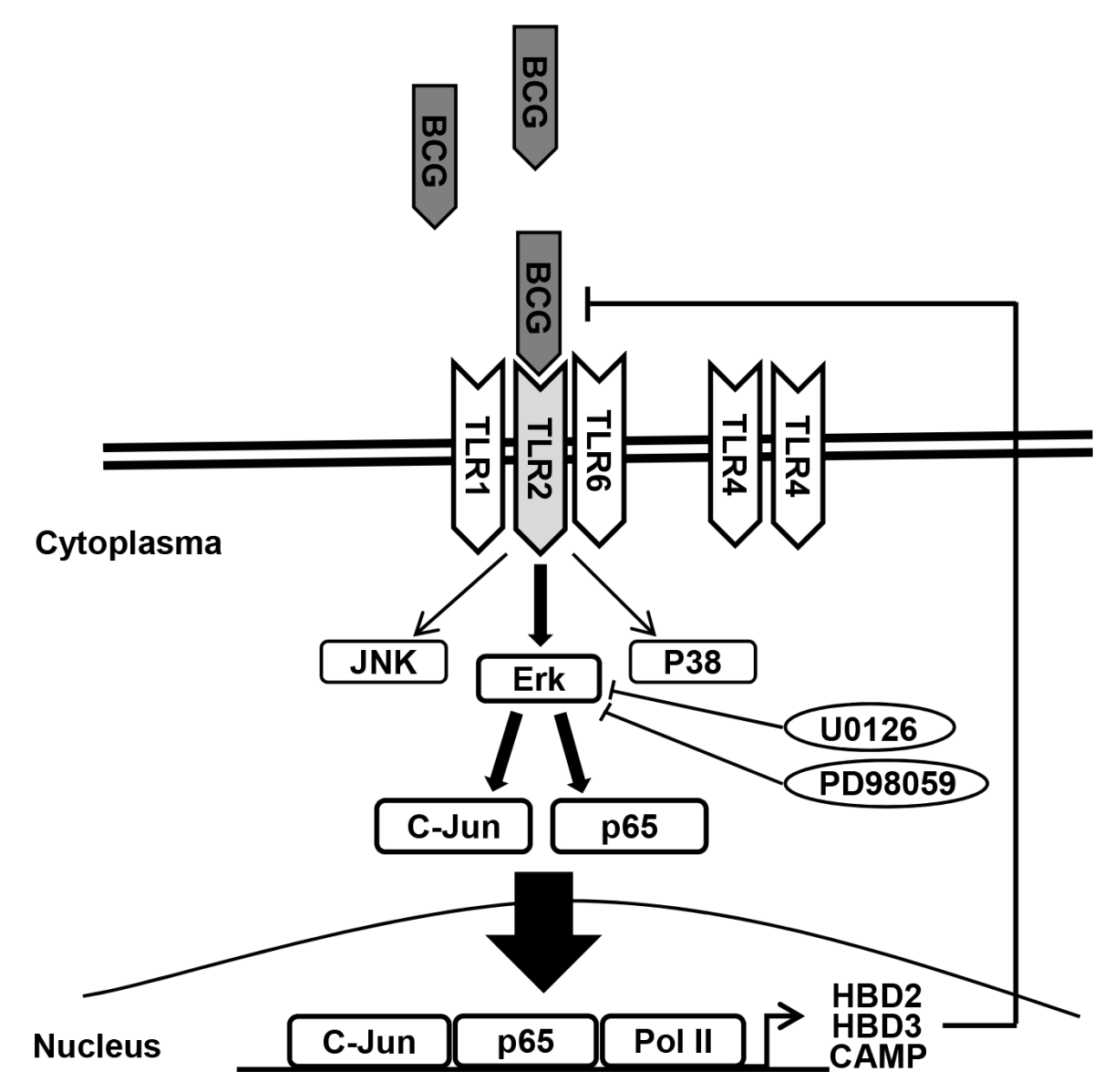

Figure 6: Schematic diagram of the working hypothesis. BCG induces ERK phosphorylation via TLR 2, leading to c-Jun- and p65-dependent transcription of HBD2, HBD3, and CAMP. Pharmacological inhibition of ERK phosphorylation and/or genetic ablation of TLR2 enhance the anti-proliferative effects of BCG on bladder cancer cells. 
[32]. The following primary antibodies were purchased from Cell Signaling Technology (MA, USA): rabbit polyclonal antibodies against phospho-extracellular signal-regulated kinase (phospho-Erk; Thr202/Tyr204), phospho-p38 mitogen-activated protein kinase (MAPK; Thr180/Tyr182), phospho-stress-activated protein kinase (SAPK)/JNK (Thr183/Tyr185), c-Jun, p65, Pol II, cleaved PARP, and mouse monoclonal antibody against phosphorylated p70S6K (Thr389). Goat polyclonal anti-actin antibody was purchased from Santa Cruz Biotechnology (CA, USA). All experiments were performed in triplicate.

\section{Chromatin immunoprecipitation (ChIP)}

ChIP analysis was performed using ChIP assay solutions (Santa Cruz Biotech, CA, USA) as described previously [33]. Briefly, shRNA TLR2 or TLR4 stable knockdown cells were treated with BCG for $8 \mathrm{~h}$ and then incubated with $10 \%$ formaldehyde to crosslink the histones to the DNA. Following preclearing, anti-c-Jun antibody, anti-p65 antibody, and anti-Pol II antibody were incubated overnight at $4^{\circ} \mathrm{C}$ with rotation. After elution, the crosslinks were reversed and the immunoprecipitated DNA was recovered by phenol/chloroform extraction and analyzed using PCR. The following primer pairs used for the ChIP analyses: HBD2 promoter (forward 5'-CAGGGTTTCTTCAGAACCTGA-3' and reverse 5'-TGAGGTCTCTGGTGTCTCTC-3'), HBD3 promoter (forward 5'- CAAAGTACGGACAAGTCAGC -3' and reverse 5'- TTCCAGCCACAGCTGCAATT -3'), CAMP promoter (forward 5'- TTGGCTTGGGAGAAGCCAT -3' and reverse 5'- AACACAGTAGCCACCCCCAA-3').

\section{Statistical analysis}

The results are presented as the mean \pm standard deviation (SD) for at least three separate experiments performed in triplicate. The significance of differences between values was determined by using the Student t-test. Two-sided $P<0.05$ was considered statistically significant [34].

\section{Author contributions}

Y.M.W. and S.B.J. performed the research; I.H.C. designed the research; Y.M.W. and S.I.P wrote the manuscript; and I.H.C. and Y.M.W analyzed the data.

\section{ACKNOWLEDGMENTS}

This work was supported by the National Research Foundation (NRF) of the Republic of Korea (2015R1A1A1A0500110 and 2015R1A2A1A15054364 to I.H.C., 2015R1C1A1A01051508 to S.I.P., and 2016R1D1A1B03933826 to Y.M.W.); Korea University Research Grant (S.I.P.); the Korea Health Technology
R\&D Project (HI17C0710 to I.H.C.); and the National Cancer Center (Grant No. 1720140 to S.I.P.), the Ministry of Health and Welfare of Korea.

\section{CONFLICTS OF INTEREST}

All authors declare no financial conflicts of interest.

\section{REFERENCES}

1. Shelley M, Court JB, Kynaston H, Wilt TJ, Fish R, Mason M. Intravesical Bacillus Calmette-Guérin in Ta and T1 bladder cancer. Cochrane Database Syst Rev. 2000; CD001986.

2. Pashos CL, Botteman MF, Laskin BL, Redaelli A. Bladder cancer: epidemiology, diagnosis, and management. Cancer Pract. 2002; 10: 311-322.

3. Elass E, Coddeville B, Kremer L, Mortuaire M, Mazurier J, Guérardel Y. Mycobacterial lipomannan induces MAP kinase phosphatase-1 expression in macrophages. FEBS Lett. 2008; 582: 445-450.

4. Yuk JM, Shin DM, Yang CS, Kim KH, An SJ, Rho J, Park JK, Jo EK. Role of apoptosis-regulating signal kinase 1 in innate immune responses by Mycobacterium bovis bacillus Calmette-Guerin. Immunol Cell Biol. 2008; 87: 100-107.

5. Uehori J, Matsumoto M, Tsuji S, Akazawa T, Takeuchi O, Akira S, Kawata T, Azuma I, Toyoshima K, Seya T. Simultaneous blocking of human Toll-like receptors 2 and 4 suppresses myeloid dendritic cell activation induced by Mycobacterium bovis bacillus Calmette-Guérin peptidoglycan. Infect Immun. 2003; 71: 4238-4249.

6. Medzhitov R, Janeway CA Jr. Innate immunity: the virtues of a nonclonal system of recognition. Cell. 1997; 91: 295-298.

7. Akira S, Takeda K, Kaisho T. Toll-like receptors: critical proteins linking innate and acquired immunity. Nature Immunol. 2001; 2: 675-680.

8. Kyriakis JM, Avruch J. Mammalian mitogen-activated protein kinase signal transduction pathways activated by stress and inflammation. Physiol Rev. 2001; 81: 807.

9. Yang CS, Lee JS, Song CH, Hur GM, Lee SJ, Tanaka S, Akira $\mathrm{S}$, Paik TH, Jo EK. Protein kinase C zeta plays an essential role for Mycobacterium tuberculosis-induced extracellular signal-regulated kinase $1 / 2$ activation in monocytes/ macrophages via Toll-like receptor 2. Cell Microbiol. 2007; 9: 382-396.

10. Ryffel B, Fremond C, Jacobs M, Parida S, Botha T, Schnyder B, Quesniaux V. Innate immunity to mycobacterial infection in mice: critical role for Toll-like receptors. Tuberculosis (Edinb). 2005; 85: 395-405.

11. Briken V, Porcelli SA, Besra GS, Kremer L. Mycobacterial lipoarabinomannan and related lipoglycans: from biogenesis to modulation of the immune response. Mol Microbiol. 2004; 53: 391-403. 
12. Selsted ME, Ouellette AJ. Mammalian defensins in the antimicrobial immune response. Nat Immunol. 2005; 6: 551-557.

13. Lehrer RI, Lichtenstein AK, Ganz T. Defensins: antimicrobial and cytotoxic peptides of mammalian cells. Annu Rev Immunol. 1993; 11: 105-128.

14. Méndez-Samperio P, Miranda E, Trejo A. Expression and secretion of cathelicidin LL-37 in human epithelial cells after infection by Mycobacterium bovis Bacillus CalmetteGuérin. Clin Vaccine Immunol. 2008; 15: 1450-1455.

15. Méndez-Samperio P, Miranda E, Trejo A. Mycobacterium bovis Bacillus Calmette-Guérin (BCG) stimulates human beta-defensin-2 gene transcription in human epithelial cells. Cell Immunol. 2006; 239: 61-66.

16. Kim JH, Kim SJ, Lee KM, Chang IH. Human $\beta$-defensin 2 may inhibit internalisation of bacillus Calmette-Guérin (BCG) in bladder cancer cells. BJU Int. 2013; 112: 781-790.

17. Méndez-Samperio P, Alba L, Trejo A. Mycobacterium bovis-mediated induction of human beta-defensin-2 in epithelial cells is controlled by intracellular calcium and p38MAPK. J Infect. 2007; 54: 469-474.

18. Ghorpade DS, Holla S, Sinha AY, Alagesan SK, Balaji KN. Nitric oxide and KLF4 protein epigenetically modify class II transactivator to repress major histocompatibility complex II expression during Mycobacterium bovis bacillus Calmette-Guerin infection. J Biol Chem. 2013; 288: 20592-20606

19. Méndez-Samperio P, Trejo A, Miranda E. Mycobacterium bovis BCG induces $\mathrm{CXC}$ chemokine ligand 8 secretion via the MEK-dependent signal pathway in human epithelial cells. Cell Immunol. 2005; 234: 9-15.

20. Schorey JS, Cooper AM. Macrophage signalling upon mycobacterial infection: the MAP kinases lead the way. Cell Microbiol. 2003; 5: 133-142.

21. An H, Yu Y, Zhang M, Xu H, Qi R, Yan X, Liu S, Wang W, Guo Z, Guo J, Qin Z, Cao X. Involvement of ERK, p38 and NF-kappaB signal transduction in regulation of TLR2, TLR4 and TLR9 gene expression induced by lipopolysaccharide in mouse dendritic cells. Immunology. 2002; 106: 38-45.

22. Kresowik TP, Griffith TS. Bacillus Calmette-Guerin immunotherapy for urothelial carcinoma of the bladder. Immunotherapy. 2009; 1: 281-288.

23. Sharma D, Tiwari BK, Mehto S, Antony C, Kak G, Singh Y, Natarajan K. Suppression of protective responses upon activation of L-type voltage gated calcium channel in macrophages during Mycobacterium bovis BCG infection. PLoS One. 2016; 11: e0163845. doi: 10.1371/journal. pone. 0163845 .

24. Krisanaprakornkit S, Kimball JR, Dale BA. Regulation of human beta-defensin-2 in gingival epithelial cells: the involvement of mitogen-activated protein kinase pathways, but not the NF-kappaB transcription factor family. J Immunol. 2002; 168: 316-324.

25. Vora P, Youdim A, Thomas LS, Fukata M, Tesfay SY, Lukasek K, Michelsen KS, Wada A, Hirayama T, Arditi M, Abreu MT. Beta-defensin-2 expression is regulated by TLR signaling in intestinal epithelial cells. J Immunol. 2004; 173: $5398-5405$

26. Darieva Z, Lasunskaia EB, Campos MN, Kipnis TL, Da Silva WD. Activation of phosphatidylinositol 3-kinase and c-Jun-N-terminal kinase cascades enhances NF- $\mathrm{kB}$ dependent gene transcription in BCG-stimulated macrophages through promotion of p65/p300 binding. J Leukoc Biol. 2004; 75: 689-697.

27. Murata M. Activation of Toll-like receptor 2 by a novel preparation of cell wall skeleton from Mycobacterium bovis BCG Tokyo (SMP-105) sufficiently enhances immune responses against tumors. Cancer Sci. 2008; 99: 1435-1440.

28. Means TK, Jones BW, Schromm AB, Shurtleff BA, Smith JA, Keane J, Golenbock DT, Vogel SN, Fenton MJ. Differential effects of a Toll-like receptor antagonist on Mycobacterium tuberculosis-induced macrophage responses. J Immunol. 2001; 166: 4074-4082.

29. Sánchez D, Rojas M, Hernández I, Radzioch D, García LF, Barrera LF. Role of TLR2- and TLR4-mediated signaling in Mycobacterium tuberculosis-induced macrophage death. Cell Immunol. 2010; 260: 128-136.

30. Park SI, Lee C, Sadler WD, Koh AJ, Jones J, Seo JW, Soki FN, Cho SW, Daignault SD, McCauley LK. Parathyroid hormone-related protein drives a $\mathrm{CD} 11 \mathrm{~b}+\mathrm{Gr} 1+$ cellmediated positive feedback loop to support prostate cancer growth. Cancer Res. 2013; 73: 6574-6583. doi: 10.1158/0008-5472.CAN-12-4692.

31. Whang YM, Park SI, Trenary IA, Egnatchik RA, Fessel JP, Kaufman JM, Carbone DP, Young JD. LKB1 deficiency enhances sensitivity to energetic stress induced by erlotinib treatment in non-small-cell lung cancer (NSCLC) cells. Oncogene. 2016; 35: 856-866.

32. Whang YM, Kim YH, Kim JS, Yoo YD. RASSF1A suppresses the c-Jun-NH2-kinase pathway and inhibits cell cycle progression. Cancer Res. 2005; 65: 3682.

33. Whang YM, Choi EJ, Seo JH, Kim JS, Yoo YD, Kim YH. Hyperacetylation enhances the growth-inhibitory effect of all-trans retinoic acid by the restoration of retinoic acid receptor $\beta$ expression in head and neck squamous carcinoma (HNSCC) cells. Cancer Chemother Pharmacol. 2005; 56: 543-555.

34. Park SI, Liao J, Berry JE, Li X, Koh AJ, Michalski ME, Eber MR, Soki FN, Sadler D, Sud S, Tisdelle S, Daignault $\mathrm{SD}$, Nemeth JA, et al. Cyclophosphamide creates a receptive microenvironment for prostate cancer skeletal metastasis. Cancer Res. 2012; 72: 2522-2532. 